\title{
On the Hausdorff Dimension of Bernoulli Convolutions
}

DOI:

10.1093/imrn/rny209

\section{Document Version}

Accepted author manuscript

Link to publication record in Manchester Research Explorer

\section{Citation for published version (APA):}

Kempton, T., Persson, T., Feng, D-J., \& Akiyama, S. (2018). On the Hausdorff Dimension of Bernoulli Convolutions. International Mathematics Research Notices, 2020(19), 6569-6595.

https://doi.org/10.1093/imrn/rny209

\section{Published in:}

International Mathematics Research Notices

\section{Citing this paper}

Please note that where the full-text provided on Manchester Research Explorer is the Author Accepted Manuscript or Proof version this may differ from the final Published version. If citing, it is advised that you check and use the publisher's definitive version.

\section{General rights}

Copyright and moral rights for the publications made accessible in the Research Explorer are retained by the authors and/or other copyright owners and it is a condition of accessing publications that users recognise and abide by the legal requirements associated with these rights.

\section{Takedown policy}

If you believe that this document breaches copyright please refer to the University of Manchester's Takedown Procedures [http://man.ac.uk/04Y6Bo] or contact uml.scholarlycommunications@manchester.ac.uk providing relevant details, so we can investigate your claim.

\section{OPEN ACCESS}




\title{
On the Hausdorff Dimension of Bernoulli Convolutions*
}

\author{
Shigeki Akiyama, De-Jun Feng, \\ Tom Kempton and Tomas Persson
}

September 4, 2018

\begin{abstract}
We give an expression for the Garsia entropy of Bernoulli convolutions in terms of products of matrices. This gives an explicit rate of convergence of the Garsia entropy and shows that one can calculate the Hausdorff dimension of the Bernoulli convolution $\nu_{\beta}$ to arbitrary given accuracy whenever $\beta$ is algebraic. In particular, if the Garsia entropy $H(\beta)$ is not equal to $\log (\beta)$ then we have a finite time algorithm to determine whether or not $\operatorname{dim}_{H}\left(\nu_{\beta}\right)=1$.
\end{abstract}

\section{Introduction}

Bernoulli convolutions are a simple and interesting family of self-similar measures with overlaps. For $\beta \in(1,2)$, the Bernoulli convolution $\nu_{\beta}$ is defined as the weak-star limit of the family of measures $\nu_{\beta}^{(n)}$ given by

$$
\nu_{\beta}^{(n)}:=\frac{1}{2^{n}} \sum_{a_{1} \cdots a_{n} \in\{0,1\}^{n}} \delta_{\sum_{i=1}^{n} a_{i} \beta^{-i}}
$$

*Accepted Author Manuscript, published in International Mathematics Research Notices https://academic.oup.com/imrn 
The fundamental questions relating to the Bernoulli convolution $\nu_{\beta}$ are whether it has Hausdorff dimension one, and if so, whether it is absolutely continuous.

Erdös proved that $\nu_{\beta}$ is singular whenever $\beta$ is a Pisot number [6], and it was later proved by Garsia that in fact $\nu_{\beta}$ has Hausdorff dimension less than one whenever $\beta$ is Pisot [14]. So far, Pisot numbers are the only class of $\beta$ for which it is known that $\nu_{\beta}$ is singular. Garsia gave a small explicit class of $\beta$ for which $\nu_{\beta}$ is absolutely continuous [13], until recently these were the only examples of Bernoulli convolutions for which it was known that the Hausdorff dimension is one. In [23] Solomyak proved that $\nu_{\beta}$ is absolutely continuous for Lebesgue-almost every $\beta \in(1,2)$.

A great deal of the recent progress on Bernoulli convolutions stems from Hochman's article [17], where it was proved that if $\nu_{\beta}$ has Hausdorff dimension less than one then the sums in the definition of $\nu_{\beta}^{(n)}$ must be superexponentially close. This can only happen on a set of $\beta$ of Hausdorff dimension zero. Additionally, Hochman proved that if $\beta$ is algebraic then $\operatorname{dim}_{H}\left(\nu_{\beta}\right)$ can be expressed in terms of the Garsia entropy of $\beta$, which will be defined in Section 1.1.

Further recent progress was made by Breuillard and Varjú [4], where it was proved that

$$
H(\beta) \geq 0.44 \min \left\{\log 2, \log M_{\beta}\right\},
$$

for any algebraic integer $\beta \in(1,2)$, where $H(\beta)$ is the Garsia entropy of $\nu_{\beta}$ (see Section 1.1 for the definition) and $M_{\beta}$ is the Mahler measure of $\beta$ defined by $M_{\beta}=\prod_{\left|\beta_{i}\right|>1}\left|\beta_{i}\right|$, where $\beta_{i}$ are the algebraic conjugates (including $\beta$ itself) of $\beta$. This implies that for an algebraic integer $\beta \in(1,2), \operatorname{dim}_{\mathrm{H}}\left(\nu_{\beta}\right)=1$ if $0.44 \min \left\{\log 2, \log M_{\beta}\right\} \geq \log \beta$ (see (1)).

In [5], Breuillard and Varjú showed, among other results, that

$$
\left\{\beta \in(1,2): \operatorname{dim}_{\mathrm{H}}\left(\nu_{\beta}\right)<1\right\} \subset \overline{\left\{\beta \in(1,2) \cap \overline{\mathbb{Q}}: \operatorname{dim}_{\mathrm{H}}\left(\nu_{\beta}\right)<1\right\}},
$$

where $\overline{\mathbb{Q}}$ is the set of algebraic numbers. This, together with Hochman's results, has sparked renewed interest in the study of Garsia entropy for algebraic parameters. If one were able to show that Pisot numbers are the only algebraic numbers corresponding to Bernoulli convolutions of dimension less than one, this would show that all non-Pisot $\beta$ give rise to Bernoulli convolutions of dimension 1 (without the restriction that $\beta$ should be algebraic). 
There have also been recent results on the absolute continuity of Bernoulli convolutions. Shmerkin [22] proved further that $\nu_{\beta}$ is absolutely continuous for all $\beta \in(1,2) \backslash \mathcal{E}$ where $\mathcal{E}$ is a set of exceptions of Hausdorff dimension zero. In [24], Varjú gave new explicit examples of absolutely continuous Bernoulli convolutions. For a recent summary of progress on Bernoulli convolutions, see $[25]$.

In this article we are interested in expressing the Garsia entropy and the dimension of Bernoulli convolutions $\nu_{\beta}$ in terms of products of matrices. There is some precedent for this, see in particular [8, 10], but these previous ideas are based on tilings of the unit interval related to the construction of $\nu_{\beta}$, and cannot be generalised to the non-Pisot cases. We use a different approach to show that, for any algebraic integer $\beta$, one can construct matrices whose products encode information about the Garsia entropy. In particular, we give a sequence of lower bounds for the Garsia entropy which yield an explicit rate of convergence in the Garsia entropy formula.

\section{$1.1 \quad$ Statement of Results}

Let $\Sigma:=\{0,1\}^{\mathbb{N}}$. For $p \in(0,1)$, let $m_{p}$ denote the $(p, 1-p)$-Bernoulli product measure on $\Sigma$ which gives weight $p$ to digit 0 and weight $1-p$ to digit 1 . For $\beta \in(1,2)$, the transformation $\pi_{\beta}: \Sigma \rightarrow \mathbb{R}$ defined by

$$
\pi_{\beta}:\left(a_{i}\right)_{i=1}^{\infty} \mapsto \sum_{i=1}^{\infty} a_{i} \beta^{-i}
$$

maps the measure $m_{p}$ to a measure $\nu_{\beta, p}$ on $\mathbb{R}$. That is, $\nu_{\beta, p}=m_{\beta} \circ \pi_{\beta}^{-1}$. For $p=\frac{1}{2}$, we get the Bernoulli convolution $\nu_{\beta}=\nu_{\beta, \frac{1}{2}}$, which was defined in the previous section. For $p \neq \frac{1}{2}$ we get a so-called biased Bernoulli convolution.

Given a word $a_{1} \cdots a_{n} \in\{0,1\}^{n}$, let the cylinder set $\left[a_{1} \cdots a_{n}\right]$ be defined by

$$
\left[a_{1} \cdots a_{n}\right]=\left\{\underline{b}=\left(b_{i}\right)_{i=1}^{\infty} \in \Sigma: b_{1} \cdots b_{n}=a_{1} \cdots a_{n}\right\} .
$$

Given a sequence $\underline{a}=\left(a_{i}\right)_{i=1}^{\infty} \in\{0,1\}^{\mathbb{N}}$, let

$$
\mathcal{N}_{n}(\underline{a})=\mathcal{N}_{n}\left(a_{1}, \ldots, a_{n}\right)=\left|\left\{\left(b_{1}, \ldots, b_{n}\right) \in\{0,1\}^{n}: \sum_{i=1}^{n} b_{i} \beta^{-i}=\sum_{i=1}^{n} a_{i} \beta^{-i}\right\}\right|
$$


and

$$
\mathcal{M}_{n}(\underline{a}, p)=\sum_{\substack{\sum_{1}^{n} b_{1} \cdots b_{n} \in\{0,1\}^{n} \\ \sum_{i=1}^{n} b_{i} \beta^{-i}=\sum_{i=1}^{n} a_{i} \beta^{-i}}} m_{p}\left[b_{1} \cdots b_{n}\right] .
$$

In what follows we write $\mathcal{M}_{n}(\underline{a})$ or $\mathcal{M}_{n}\left(a_{1} \cdots a_{n}\right)$, de-emphacising the dependence on $p$ since we consider $p$ to be fixed.

Let $^{1}$

$$
H_{n}(\beta, p):=-\sum_{a_{1} \cdots a_{n} \in\{0,1\}^{n}} m_{p}\left[a_{1} \cdots a_{n}\right] \log \mathcal{M}_{n}\left(a_{1} \cdots a_{n}, p\right) .
$$

Finally we let

$$
H(\beta, p):=\lim _{n \rightarrow \infty} \frac{1}{n} H_{n}(\beta, p) .
$$

$H(\beta, p)$ is called the Garsia entropy ${ }^{2}$ of $\nu_{\beta, p}$. In particular, we write $H_{n}(\beta)=$ $H_{n}(\beta, 1 / 2)$ and $H(\beta)=H(\beta, 1 / 2)$.

Hochman [17] proved that if $\beta \in(1,2)$ is an algebraic number then the dimension of the Bernoulli convolution $\nu_{\beta, p}$ is given by

$$
\operatorname{dim}_{\mathrm{H}}\left(\nu_{\beta, p}\right)=\min \left\{\frac{H(\beta, p)}{\log \beta}, 1\right\}
$$

see also [4] for a more detailed explanation.

In this article we are concerned with lower bounds for $H(\beta, p)$, and hence lower bounds for $\operatorname{dim}_{\mathrm{H}}\left(\nu_{\beta, p}\right)$, when $\beta$ is an algebraic number. If $\beta$ is not an algebraic integer, i.e. not the root of a polynomial with integer coefficients where the leading coefficient is 1 , then $H(\beta, p)=\log 2$. Thus we may restrict our interest to algebraic integers.

Given an algebraic integer $\beta=\beta^{(1)}$ of degree $d$, let $\beta^{(2)}, \ldots, \beta^{(d)}$ denote its Galois conjugates, ordered by decreasing absolute value.

\footnotetext{
${ }^{1}$ It would be more standard to write

$$
H_{n}(\beta, p)=\sum_{x} \mathcal{M}_{n}^{*}(x, p) \log \mathcal{M}_{n}^{*}(x, p),
$$

where the sum is over all $x$ having a representation $x=\sum_{i=1}^{n} a_{i} \beta^{-i}$ and $\mathcal{M}_{n}^{*}(x, p)$ is just $\mathcal{M}_{n}(\underline{a}, p)$ for any $\underline{a}$ with $x=\sum_{i=1}^{n} a_{i} \beta^{-i}$. These expressions are clearly equivalent, we find ours more convenient since we work only with sequences and since the above makes the link with Lyapunov exponents of pairs of matrices more direct.

${ }^{2}$ Beware, there are two different conventions for the definition of Garsia entropy. Some authors divide by $\log (\beta)$ in the definition.
} 
Theorem 1.1. Let $\beta$ be an algebraic integer of degree $d$ and let $p \in(0,1)$. The Garsia entropy $H(\beta, p)$ can be approximated with explicit error bounds. In particular,

$$
\frac{1}{n} H_{n}(\beta, p)-\frac{C+l \log (n+1)}{n} \leq H(\beta, p) \leq \frac{1}{n} H_{n}(\beta, p)
$$

for all $n \in \mathbb{N}$, where

$$
C=\log \left(2^{d} \prod_{i:\left|\beta^{(i)}\right| \neq 1} \frac{1}{|| \beta^{(i)}|-1|}+1\right)
$$

and $l$ is the number of conjugates of $\beta$ of absolute value 1 .

Theorem 1.1 is proved by giving lower bounds for $H(\beta, p)$ in terms of products of matrices.

Theorem 1.2. There exists a pair of non-negative matrices $M_{0}=M_{0}(\beta, p)$ and $M_{1}=M_{1}(\beta, p)$, with rows and columns indexed by a set $\mathcal{A}$, such that the sequence

$$
\frac{1}{n} L_{n}(\beta, p):=-\frac{1}{n} \sup _{i \in \mathcal{A}} \sum_{a_{1} \cdots a_{n} \in\{0,1\}^{n}} m_{p}\left[a_{1} \cdots a_{n}\right] \log \left(\sum_{j \in \mathcal{A}}\left(M_{a_{1}} \cdots M_{a_{n}}\right)_{i, j}\right)
$$

converges to $H(\beta, p)$ from below as $n \rightarrow \infty$, and $\frac{1}{n} L_{n}(\beta, p) \leq H(\beta, p)$.

The set $\mathcal{A}$ is finite (with size bounded by $C(\beta)$ given by (2)) whenever $\beta$ is hyperbolic, i.e. when it has no Galois conjugates of modulus one. In this case the matrices $M_{0}, M_{1}$ are computable by a finite time algorithm. If $\beta$ is not hyperbolic then $\mathcal{A}$ might be countably infinite, but the matrices $M_{0}, M_{1}$ have at most two non-zero terms in any row.

Theorems 1.1 and 1.2 are proved by bounding the difference between $H_{n}(\beta, p)$ and $L_{n}(\beta, p)$. When $\beta$ is hyperbolic, and so $\mathcal{A}$ is finite, Theorem 1.2 can be expressed in the more familiar form of the Lyapunov exponent of the pair of matrices $M_{0}, M_{1}$.

Theorem 1.3. When $\beta$ is hyperbolic, the sequence

$$
\frac{1}{n} L_{n}^{\prime}(\beta, p):=-\frac{1}{n} \sum_{a_{1} \cdots a_{n} \in\{0,1\}^{n}} m_{p}\left[a_{1} \cdots a_{n}\right] \log \left(\left\|M_{a_{1}} \cdots M_{a_{n}}\right\|\right)
$$

converges to $H(\beta, p)$ as $n \rightarrow \infty$, and $\frac{1}{n} L_{n}^{\prime}(\beta, p) \leq \frac{1}{n} L_{n}(\beta, p) \leq H(\beta, p)$. Here, the norm that we use is the $\ell^{\infty}$ operator norm given by $\|B\|=\sup _{i \in \mathcal{A}} \sum_{j \in \mathcal{A}}\left|B_{i j}\right|$. 
An immediate corollary is that we can express the Garsia entropy as the Lyapunov exponent of the matrices $M_{0}, M_{1}$ associated with the $(p, 1-p)$ Bernoulli product measure.

Corollary 1.4. If $\beta$ is hyperbolic then the Garsia entropy $H(\beta, p)$ is the limit of the sequence

$$
-\frac{1}{n} \log \left\|M_{a_{1}} \cdots M_{a_{n}}\right\|
$$

for $m_{p}$-a.e. $\underline{a} \in\{0,1\}^{\mathbb{N}}$.

That Corollary 1.4 follows from Theorem 1.3 is an immediate application of the Furstenberg-Kesten theorem on random matrices [12].

\section{Preliminary Results}

In this section we recall some standard algebraic lemmas as well as ideas about separation of polynomials originating in the work of Garsia [13].

Let $\beta=\beta^{(1)} \in(1,2)$ be an algebraic integer of degree $d$. Let $\beta^{(2)}, \ldots, \beta^{(r)}$ denote the algebraic conjugates of $\beta$ of modulus strictly larger than one, $\beta^{(r+1)}, \ldots, \beta^{(r+l)}$ conjugates of modulus 1 , and $\beta^{(r+l+1)}, \ldots, \beta^{(d)}$ conjugates of modulus less than one.

The following lemmas are standard.

Lemma 2.1. If $\sum_{i=1}^{n} \epsilon_{i} \beta^{-i}=0$ for $\epsilon_{i} \in\{-1,0,1\}$ then

$$
\sum_{i=1}^{n} \epsilon_{i}\left(\beta^{(j)}\right)^{-i}=0
$$

for each $j \in\{2, \ldots, d\}$.

Lemma 2.2. Let $P$ be a polynomial with integer coefficients. Then the product $P(\beta) P\left(\beta^{(2)}\right) \cdots P\left(\beta^{(d)}\right)$ is an integer.

Note that this second lemma requires that $\beta$ is an algebraic integer, i.e. the root of a polynomial with integer coefficients whose leading term is 1 . It does not hold for all algebraic numbers. 
For $n \geq 0$, define the set $V_{\beta, n} \subset\left[\frac{-1}{\beta-1}, \frac{1}{\beta-1}\right]$ by

$$
\begin{aligned}
V_{\beta, n}:=\{x & =\sum_{i=0}^{n} \epsilon_{i} \beta^{n-i}: \epsilon_{i} \in\{-1,0,1\} \\
& \text { and } \left.\left|\sum_{i=0}^{n} \epsilon_{i}\left(\beta^{(j)}\right)^{n-i}\right| \leq \frac{1}{\left|\beta^{(j)}\right|-1} \text { for all } j \in\{1, \ldots r\}\right\} .
\end{aligned}
$$

Clearly, $V_{\beta, n} \subset V_{\beta, n+1}$. Let

$$
V_{\beta}:=\bigcup_{n=0}^{\infty} V_{\beta, n}
$$

Lemma 2.3. Suppose that $\sum_{i=0}^{n} \epsilon_{i} \beta^{n-i}=0$ for some $n \geq 1$. Then

$$
\sum_{i=0}^{m} \epsilon_{i} \beta^{m-i} \in V_{\beta, m} \subset V_{\beta}
$$

for each $m \in\{0, \ldots, n\}$.

Proof. Suppose on the contrary that

$$
\sum_{i=0}^{m} \epsilon_{i} \beta^{m-i} \notin V_{\beta} \quad \text { for some } m \in\{0,1, \ldots, n-1\} .
$$

Then by definition, there exists $j \in\{1, \ldots, r\}$ such that

$$
\left|\sum_{i=0}^{m} \epsilon_{i}\left(\beta^{(j)}\right)^{m-i}\right|>\frac{1}{\left|\beta^{(j)}\right|-1}
$$

Then

$$
\begin{aligned}
\left|\sum_{i=0}^{m+1} \epsilon_{i}\left(\beta^{(j)}\right)^{m+1-i}\right| & =\left|\epsilon_{m+1}+\beta^{(j)} \sum_{i=0}^{m} \epsilon_{i}\left(\beta^{(j)}\right)^{m-i}\right| \\
& \geq\left|\beta^{(j)}\right|\left|\sum_{i=0}^{m} \epsilon_{i}\left(\beta^{(j)}\right)^{m-i}\right|-1 \\
& >\frac{\left|\beta^{(j)}\right|}{\left|\beta^{(j)}\right|-1}-1=\frac{1}{\left|\beta^{(j)}\right|-1} .
\end{aligned}
$$


Iterating this argument gives that

$$
\left|\sum_{i=0}^{n} \epsilon_{i}\left(\beta^{(j)}\right)^{n-i}\right|>\frac{1}{\left|\beta^{(j)}\right|-1} .
$$

But by Lemma 2.1 the quantity on the left hand side is equal 0 , since $\sum_{i=0}^{n} \epsilon_{i} \beta^{n-i}=0$. This gives a contradiction.

Let

$$
C(\beta):=2^{d} \prod_{j=1}^{r} \frac{1}{\left|\beta^{(j)}\right|-1} \prod_{k=r+l+1}^{d} \frac{1}{1-\left|\beta^{(k)}\right|}=2^{d} \prod_{\left|\beta^{(j)}\right| \neq 1} \frac{1}{|| \beta^{(j)}|-1|} .
$$

This is a product over all roots which do not have modulus one. The following lemma is essentially due to Garsia, see also [11, 2, 20].

Lemma 2.4. For $n \geq 0$, we have

$$
\left|V_{\beta, n}\right| \leq C(\beta)(n+1)^{l}+1
$$

In particular, if $\beta$ is hyperbolic then $V_{\beta}$ is finite.

Proof. Fix $n \geq 0$. Let $V_{\beta, n}^{\prime} \subset\left[\frac{-2}{\beta-1}, \frac{2}{\beta-1}\right]$ be given by

$$
\begin{aligned}
V_{\beta, n}^{\prime}:=\left\{x=\sum_{i=0}^{n} \epsilon_{i} \beta^{n-i}: \epsilon_{i} \in\{-2,-1,0,1,2\}\right. \\
\left.\quad \text { and }\left|\sum_{i=0}^{n} \epsilon_{i}\left(\beta^{(j)}\right)^{n-i}\right| \leq \frac{2}{\left|\beta^{(j)}\right|-1} \text { for all } j \in\{1, \ldots r\}\right\} .
\end{aligned}
$$

For a non-zero $x \in V_{\beta, n}^{\prime}$, given by

$$
x=\sum_{i=0}^{n} \epsilon_{i} \beta^{n-i}
$$

with $\epsilon_{i} \in\{-2,-1,0,1,2\}$, write

$$
x^{(j)}=\sum_{i=0}^{n} \epsilon_{i}\left(\beta^{(j)}\right)^{n-i}, \quad j=1, \ldots, d .
$$


Then

$$
\prod_{j=1}^{d}\left|x^{(j)}\right| \geq 1
$$

since $\prod_{j=1}^{d} x^{(j)}$ is an integer, which is non-zero as $x \neq 0$.

Now for $j \in\{r+l+1, \ldots, d\}$,

$$
\left|x^{(j)}\right|=\left|\sum_{i=0}^{n} \epsilon_{i}\left(\beta^{(j)}\right)^{n-i}\right| \leq \sum_{i=0}^{n} 2\left|\beta^{(j)}\right|^{n-i} \leq \frac{2}{1-\left|\beta^{(j)}\right|} .
$$

Furthermore, for $j \in\{2, \ldots, r\}$,

$$
\left|x^{(j)}\right| \leq \frac{2}{\left|\beta^{(j)}\right|-1},
$$

since $x \in V_{\beta, n}^{\prime}$.

Finally, for $j \in\{r+1, \cdots, r+l\}$,

$$
\left|x^{(j)}\right|=\left|\sum_{i=0}^{n} \epsilon_{i}\left(\beta^{(j)}\right)^{n-i}\right| \leq \sum_{i=0}^{n} 2 \cdot 1^{n-i} \leq 2(n+1) .
$$

Then by (3),

$$
|x| \geq \frac{1}{\prod_{i \in\{2, \ldots, d\}}\left|x^{(i)}\right|} \geq C_{0}(n)
$$

where

$$
C_{0}(n):=2^{-(d-1)}\left(\prod_{j \in\{2, \ldots, r\}}\left(\left|\beta^{(j)}\right|-1\right)\right) \frac{1}{(n+1)^{l}}\left(\prod_{j \in\{r+1, \ldots, d\}}\left(1-\left|\beta^{(j)}\right|\right)\right) .
$$

Hence, any $x \in V_{\beta, n}^{\prime} \backslash\{0\}$ has modulus at least $C_{0}(n)$.

Then for $y, z \in V_{\beta, n}$ with $y \neq z$, we have

$$
0 \neq y-z \in V_{\beta, n}^{\prime}
$$

Hence $|y-z| \geq C_{0}(n)$. This shows that any two different elements of $V_{\beta, n}$ are separated by at least $C_{0}(n)$. Therefore, since $V_{\beta, n} \subset[-1 /(\beta-1), 1 /(\beta-1)]$, this shows that $V_{\beta, n}$ contains at most

$$
\frac{2}{\beta-1} \frac{1}{C_{0}(n)}+1=C(\beta)(n+1)^{l}+1
$$

elements. 


\section{Matrices, Lyapunov Exponents, and Lower Bounds for Garsia Entropy}

We now show how the sets $V_{\beta, n}$ of the previous section have a natural graph structure, which allows one to compute lower bounds for Garsia entropy.

Start with the sets $V_{\beta, 0}=\{1,0,-1\}$ and $A_{0}=\{1,0,-1\}$. At stage $n \geq 1$ we let $V_{\beta, n}=V_{\beta, n-1} \bigcup A_{n}$ where

$$
A_{n}=\left\{\beta x-\epsilon_{n}: \epsilon_{n} \in\{-1,0,1\}, x \in A_{n-1}, \beta x-\epsilon_{n} \in V_{\beta}\right\} .
$$

If $\beta$ is hyperbolic, we stop the algorithm at the stage $n$ for which $V_{\beta, n}=$ $V_{\beta, n-1}$. Since in the hyperbolic case $V_{\beta}$ is finite, the algorithm must stop in finite time with $V_{\beta, n}=V_{\beta}$. If $\beta$ is not hyperbolic then $V_{\beta}$ may be countably infinite, but $V_{\beta, n}$ grows at most polynomially in $n$.

For each $x, y \in V_{\beta}$, draw a directed edge from $x$ to $y$, labelled by $\epsilon \in$ $\{-1,0,1\}$, whenever $y=\beta x+\epsilon$. Call the resulting graph $\mathcal{G}$.

There is a simple connection between the graph $\mathcal{G}$ and the quantities $\mathcal{N}_{n}(\underline{a})$.

Suppose that for $\underline{a}=\left(a_{i}\right)_{i=1}^{\infty}$ and $\underline{b}=\left(b_{i}\right)_{i=1}^{\infty}$ we have

$$
\sum_{i=1}^{n} a_{i} \beta^{-i}=\sum_{i=1}^{n} b_{i} \beta^{-i}
$$

Then, by the definition of $V_{\beta}$ and Lemma 2.3, for each $m \in\{1, \ldots, n\}$ we have

$$
d_{m}(\underline{a}, \underline{b}):=\beta^{m} \sum_{i=1}^{m}\left(a_{i}-b_{i}\right) \beta^{-i} \in V_{\beta} .
$$

Then, letting $d_{0}(\underline{a}, \underline{b}):=0$, we see that the word $d_{0}(\underline{a}, \underline{b}) d_{1}(\underline{a}, \underline{b}) \cdots d_{n}(\underline{a}, \underline{b})$ follows a path from 0 to 0 on the graph $\mathcal{G}$, following at each step $i$ an edge labelled by $\left(a_{i}-b_{i}\right) \in\{-1,0,1\}$.

Given a word $a_{1} \cdots a_{n} \in\{0,1\}^{n}$ and $\epsilon_{1} \cdots \epsilon_{n} \in\{-1,0,1\}^{n}$, we write

$$
\epsilon_{1} \cdots \epsilon_{n} \sim a_{1} \cdots a_{n}
$$

if $a_{i}-\epsilon_{i} \in\{0,1\}$ for each $i \in\{1, \ldots, n\}$. Then

$$
\begin{aligned}
& \mathcal{N}_{n}(\underline{a})=\mid\left\{\epsilon_{1} \cdots \epsilon_{n} \sim a_{1} \cdots a_{n}\right. \text { such that there is a path } \\
& \left.\quad \text { from } 0 \text { to } 0 \text { in } \mathcal{G} \text { obtained by following the edges } \epsilon_{1} \cdots \epsilon_{n}\right\} \mid .
\end{aligned}
$$


We can write down matrices which encode the choices of move $\epsilon_{i}$ available given $a_{i}$.

Let $x_{1}, x_{2}, \ldots$ be some ordering of the elements of $V_{\beta}$, with $x_{1}=0$. Let $\mathcal{A}=\left\{1, \ldots,\left|V_{\beta}\right|\right\}$ if $V_{\beta}$ is finite, and $\mathbb{N}$ otherwise. We want to write down matrices $M_{0}$ and $M_{1}$ such that, for a word $a_{1} \cdots a_{n}$,

$$
\left(M_{a_{1}} \cdots M_{a_{n}}\right)_{i, j}=\sum_{\substack{b_{1} \cdots b_{n} \in\{0,1\}^{n} \\ \beta^{n} x_{i}+\sum_{l=1}^{n}\left(a_{l}-b_{l}\right) \beta^{n-l}=x_{j}}} m_{p}\left[b_{1} \cdots b_{n}\right] .
$$

Let $M_{0}$ be the $\left|V_{\beta}\right| \times\left|V_{\beta}\right|$ matrix such that

$$
\left(M_{0}\right)_{i, j}=\left\{\begin{array}{ll}
1-p & \text { if } x_{j}=\beta x_{i}-1 \\
p & \text { if } x_{j}=\beta x_{i} \\
0 & \text { otherwise }
\end{array},\right.
$$

and let $M_{1}$ be the $\left|V_{\beta}\right| \times\left|V_{\beta}\right|$ matrix such that

$$
\left(M_{1}\right)_{i, j}=\left\{\begin{array}{ll}
1-p & \text { if } x_{j}=\beta x_{i} \\
p & \text { if } x_{j}=\beta x_{i}+1 \\
0 & \text { otherwise }
\end{array} .\right.
$$

Lemma 3.1. 1. For $x_{i}, x_{j} \in V_{\beta}$,

$$
\left(M_{a_{1}} \cdots M_{a_{n}}\right)_{i j}=\sum_{\substack{b_{1} \ldots b_{n} \in\{0,1\}^{n} \\ \beta^{n} x_{i}+\sum_{l=1}^{n}\left(a_{l}-b_{l}\right) \beta^{n-l}=x_{j}}} m_{p}\left[b_{1} \cdots b_{n}\right] .
$$

2. If $\sum_{i=1}^{n} a_{i} \beta^{-i}=\sum_{i=1}^{n} c_{i} \beta^{-i}$ for some $a_{1} \cdots a_{n}, c_{1} \cdots c_{n} \in\{0,1\}^{n}$, then

$$
M_{a_{1}} \cdots M_{a_{n}}=M_{c_{1}} \cdots M_{c_{n}} .
$$

Proof. Part 1 follows immediately from the definition of $M_{0}$ and $M_{1}$, and part 2 follows directly from part 1.

In particular,

$$
\mathcal{M}_{n}(\underline{a})=\left(M_{a_{1}} M_{a_{2}} \cdots M_{a_{n}}\right)_{11},
$$

and so we have immediately that

$$
H_{n}(\beta, p)=-\sum_{a_{1} \cdots a_{n} \in\{0,1\}^{n}} m_{p}\left[a_{1} \cdots a_{n}\right] \log \left(\left(M_{a_{1}} M_{a_{2}} \cdots M_{a_{n}}\right)_{11}\right) .
$$

As $H_{n}(\beta, p)$ is subadditive, $\frac{1}{n} H_{n}(\beta, p)$ forms a sequence which converges to $H(\beta, p)$. We have $H(\beta, p) \leq \frac{1}{n} H_{n}(\beta, p)$. 


\subsection{Lower Bounds}

For an algebraic integer $\beta$, we define

$$
\begin{aligned}
L_{n}(\beta, p): & =-\sup _{i \in \mathcal{A}} \sum_{a_{1} \cdots a_{n} \in\{0,1\}^{n}} m_{p}\left[a_{1} \cdots a_{n}\right] \log \left(\sum_{j \in \mathcal{A}}\left(M_{a_{1}} \cdots M_{a_{n}}\right)_{i, j}\right) \\
& =-\sup _{i \in \mathcal{A}} \int_{\underline{a} \in\{0,1\}^{\mathbb{N}}} \log \left(\sum_{j \in \mathcal{A}}\left(M_{a_{1}} \cdots M_{a_{n}}\right)_{i, j}\right) d m(\underline{a}) .
\end{aligned}
$$

Since

$$
M_{11} \leq \sum_{j \in \mathcal{A}} M_{1 j}
$$

for any non-negative matrix $M$, we have, by choosing $i=1$ in the above definition, that $L_{n}(\beta, p) \leq H_{n}(\beta, p)$. Here, and in much of what follows, we note the minus in the definition of $H_{n}(\beta, p)$ and $L_{n}(\beta, p)$ which reverses a lot of inequalities.

\section{Lemma 3.2.}

$$
L_{n+m}(\beta, p) \geq L_{n}(\beta, p)+L_{m}(\beta, p) \text {. }
$$

Proof. For $i \in \mathcal{A}, \underline{a} \in \Sigma$ we have

$$
\begin{aligned}
\sum_{j \in \mathcal{A}}\left(M_{a_{1}} \cdots M_{a_{n+m}}\right)_{i, j} & =\sum_{j \in \mathcal{A}} \sum_{k \in \mathcal{A}}\left(M_{a_{1}} \cdots M_{a_{n}}\right)_{i, k}\left(M_{a_{n+1}} \cdots M_{a_{n+m}}\right)_{k, j} \\
& =\sum_{k \in \mathcal{A}}\left(M_{a_{1}} \cdots M_{a_{n}}\right)_{i, k}\left(\sum_{j \in \mathcal{A}}\left(M_{a_{n+1}} \cdots M_{a_{n+m}}\right)_{k, j}\right) \\
& \leq \sum_{k \in \mathcal{A}}\left(M_{a_{1}} \cdots M_{a_{n}}\right)_{i, k}\left(\sup _{l \in \mathcal{A}} \sum_{j \in \mathcal{A}}\left(M_{a_{n+1}} \cdots M_{a_{n+m}}\right)_{l, j}\right) .
\end{aligned}
$$

It follows that

$$
\begin{aligned}
L_{n+m}(\beta, p)=-\sup _{i \in \mathcal{A}} \int_{\underline{a} \in\{0,1\}^{\mathbb{N}}} \log \left(\sum_{j \in \mathcal{A}}\left(M_{a_{1}} \cdots M_{a_{n+m}}\right)_{i, j}\right) d m(\underline{a}) \\
\geq-\sup _{i \in \mathcal{A}} \sup _{l \in \mathcal{A}} \int_{\underline{a} \in\{0,1\}^{\mathbb{N}}} \int_{\underline{b} \in\{0,1\}^{\mathbb{N}}}\left(\log \left(\sum_{k \in \mathcal{A}}\left(M_{a_{1}} \cdots M_{a_{n}}\right)_{i, k}\right)\right. \\
\left.\quad+\log \left(\sum_{j \in \mathcal{A}}\left(M_{b_{1}} \cdots M_{b_{m}}\right)_{l, j}\right)\right) d m(\underline{a}) d m(\underline{b}) \\
=L_{n}(\beta, p)+L_{m}(\beta, p) .
\end{aligned}
$$


Proposition 3.3. Let $\beta \in(1,2)$ be an algebraic integer. Then the sequence $\left(\frac{1}{n} L_{n}(\beta, p)\right)$ satisfies

$$
\frac{1}{n} H_{n}(\beta, p)-\frac{1}{n} \log \left(C(\beta)(n+1)^{l}+1\right) \leq \frac{1}{n} L_{n}(\beta, p) \leq H(\beta, p) \leq \frac{1}{n} H_{n}(\beta, p) .
$$

Proof. We have proved that $L_{n}(\beta, p)$ is superadditive. Since $H_{n}(\beta, p)$ is subadditive, $\frac{1}{n} H_{n}(\beta, p)$ converges to $H(\beta, p)$ and $\frac{1}{n} H_{n}(\beta, p) \geq \frac{1}{n} L_{n}(\beta, p)$, we see that

$$
H(\beta, p) \in\left(\frac{1}{n} L_{n}(\beta, p), \frac{1}{n} H_{n}(\beta, p)\right)
$$

for all $n \in \mathbb{N}$. Hence we need only to prove the left hand inequality.

Let

$$
X_{n}:=\left\{\sum_{i=1}^{n} a_{i} \beta^{-i}: a_{i} \in\{0,1\}\right\} .
$$

For $x \in X_{n}$ let $M_{x, n}:=M_{a_{1}} \cdots M_{a_{n}}$ for any of the words $a_{1} \cdots a_{n}$ for which

$$
x=\sum_{i=1}^{n} a_{i} \beta^{-i}
$$

This is well defined due to Lemma 3.1. Now

$$
\begin{aligned}
L_{n}(\beta, p):= & -\sup _{i \in \mathcal{A}} \sum_{a_{1} \cdots a_{n} \in\{0,1\}^{n}} m_{p}\left[a_{1} \cdots a_{n}\right] \log \left(\sum_{j \in \mathcal{A}}\left(M_{a_{1}} \cdots M_{a_{n}}\right)_{i, j}\right) \\
= & -\sup _{i \in \mathcal{A}} \sum_{x \in X_{n}}\left(M_{x, n}\right)_{1,1} \log \left(\sum_{j \in \mathcal{A}}\left(M_{x, n}\right)_{i, j}\right) \\
= & -\sum_{x \in X_{n}}\left(M_{x, n}\right)_{1,1} \log \left(\left(M_{x, n}\right)_{1,1}\right) \\
& -\sup _{i \in \mathcal{A}} \sum_{x \in X_{n}}\left(M_{x, n}\right)_{1,1} \log \left(\frac{\sum_{j \in \mathcal{A}}\left(M_{x, n}\right)_{i, j}}{\left(M_{x, n}\right)_{1,1}}\right) .
\end{aligned}
$$

The first term here is $H_{n}(\beta, p)$. Since $\sum_{x \in X_{n}}\left(M_{x, n}\right)_{1,1}=1$, we move this inside the log in the second term, and using the concavity of log we get

$$
L_{n}(\beta, p) \geq H_{n}(\beta, p)-\sup _{i \in \mathcal{A}} \log \left(\sum_{x \in X_{n}} \sum_{j \in \mathcal{A}}\left(M_{x, n}\right)_{i, j}\right) .
$$


Now recall that $\left(M_{x, n}\right)_{i, j}$ counts, for any $a_{1} \cdots a_{n}$ such that $\sum_{l=1}^{n} a_{l} \beta^{-l}=x$, the total measure of the words $b_{1} \cdots b_{n}$ for which

$$
\beta^{n} x_{i}+\sum_{l=1}^{n}\left(a_{l}-b_{l}\right) \beta^{n-l}=x_{j} .
$$

This can be rewritten as

$$
\beta^{n} x_{i}+\beta^{n} x-\sum_{l=1}^{n} b_{l} \beta^{n-l}=x_{j}
$$

In order to sum this over all $x \in X_{n}$ and $j \in \mathcal{A}$, we count for each $b_{1} \cdots b_{n} \in$ $\{0,1\}^{n}$ the number of $x \in X_{n}$ for which an equation of the form (5) is satisfied. This gives

$$
\begin{aligned}
\sum_{x \in X_{n}} \sum_{j \in \mathcal{A}}\left(M_{x, n}\right)_{i, j} & =\sum_{x \in X_{n}} \sum_{j \in \mathcal{A}} \sum_{\substack{b_{1} \cdots b_{n} \in\{0,1\}^{n} \\
(5) \text { holds }}} m_{p}\left[b_{1} \cdots b_{n}\right] \\
& =\sum_{b_{1} \cdots b_{n} \in\{0,1\}^{n}} m_{p}\left[b_{1} \cdots b_{n}\right] \cdot\left|X_{n}\left(i, b_{1} \cdots b_{n}\right)\right|
\end{aligned}
$$

where

$$
X_{n}\left(i, b_{1} \cdots b_{n}\right)=\left\{x \in X_{n}: \beta^{n} x_{i}+\left(\beta^{n} x-\sum_{l=1}^{n} b_{l} \beta^{n-l}\right) \in V_{\beta}\right\} .
$$

But now the separation arguments of Lemmma 2.4 give that, for a fixed $i$ and $b_{1} \cdots b_{n}$, sums of the form $\beta^{n} x-\sum_{l=1}^{n} b_{l} \beta^{n-l}$ are separated by at least $C_{0}(n)$ unless they are equal. This bounds the number of elements of $X_{n}\left(i, b_{1} \cdots b_{n}\right)$. Indeed, all possible values of

$$
\beta^{n} x-\sum_{l=1}^{n} b_{l} \beta^{n-l}, \quad x \in X_{n}\left(i, b_{1} \cdots b_{n}\right),
$$

are contained in the interval $\left[-\beta^{n} x_{i}-\frac{1}{\beta-1},-\beta^{n} x_{i}+\frac{1}{\beta-1}\right]$ and they are separated by at least $C_{0}(n)$. Hence $\beta^{n} x-\sum_{l=1}^{n} b_{l} \beta^{n-l}$ may attain at most

$$
\frac{2}{\beta-1} \frac{1}{C_{0}(n)}+1
$$


different values, that is

$$
\left|X_{n}\left(i, b_{1} \cdots b_{n}\right)\right| \leq \frac{2}{\beta-1} \frac{1}{C_{0}(n)}+1=C(\beta)(n+1)^{l}+1
$$

Thus

$$
\begin{aligned}
\sum_{x \in X_{n}} \sum_{j \in \mathcal{A}}\left(M_{x, n}\right)_{i, j} & =\sum_{b_{1} \cdots b_{n} \in\{0,1\}^{n}} m_{p}\left[b_{1} \cdots b_{n}\right]\left|X_{n}\left(i, b_{1} \cdots b_{n}\right)\right| \\
& \leq\left(C(\beta)(n+1)^{l}+1\right)
\end{aligned}
$$

and so

$$
L_{n}(\beta, p) \geq H_{n}(\beta, p)-\log \left(C(\beta)(n+1)^{l}+1\right)
$$

This completes the proof of both Theorem 1.1 and 1.2.

As pointed out by one of the referees, Proposition 3.3 has the following interesting consequence.

Corollary 3.4. Let $\beta \in(1,2)$ be an algebraic number. Then $H(\beta, p)$ is continuous in $p$, and so is $\operatorname{dim}_{\mathrm{H}} \nu_{\beta, p}$.

It arises a natural question whether $\operatorname{dim}_{\mathrm{H}} \nu_{\beta, p}$ is continuous in $p$ for every real $\beta \in(1,2)$. To our best knowledge, the question is open. Nevertheless, it is known that $\operatorname{dim}_{\mathrm{H}} \nu_{\beta, p}$ is always lower semi-continuous in $(\beta, p)$ (see e.g. [18, Theorem 1.8]).

\subsection{A matrix form for the hyperbolic case}

We briefly comment on two alternative lower bounds which work for the hyperbolic case and are much easier to work with. Let

$$
L_{n}^{\prime}(\beta, p):=-\sum_{a_{1} \cdots a_{n} \in\{0,1\}^{n}} m_{p}\left[a_{1} \cdots a_{n}\right] \log \left(\left\|M_{a_{1}} \cdots M_{a_{n}}\right\|\right) .
$$

$L_{n}^{\prime}(\beta, p)$ differs from $L_{n}(\beta, p)$ in that the supremum over $i \in \mathcal{A}$ happens inside the summation. Thus $L_{n}^{\prime}(\beta, p) \leq L_{n}(\beta, p)$.

Lemma 3.5. When the set $V_{\beta}$ is finite, we have

$$
H(\beta, p)=\lim _{n \rightarrow \infty} \frac{1}{n} L_{n}^{\prime}(\beta, p) .
$$


Proving this lemma completes the proof of Theorem 1.3.

Proof. Following the proof of Proposition 3.3 (or simply applying Lemma 9.9 in [26]) gives exactly

$$
L_{n}^{\prime}(\beta, p) \geq H_{n}(\beta, p)-\log \left(\sum_{x \in X_{n}}\left\|M_{x, n}\right\|\right) .
$$

But

$$
\begin{aligned}
-\log \left(\sum_{x \in X_{n}}\left\|M_{x, n}\right\|\right) & =-\log \left(\sum_{x \in X_{n}} \max _{i \in \mathcal{A}} \sum_{j \in \mathcal{A}}\left(M_{x, n}\right)_{i, j}\right) \\
& \geq-\log \left(\sum_{x \in X_{n}} \sum_{i \in \mathcal{A}} \sum_{j \in \mathcal{A}}\left(M_{x, n}\right)_{i, j}\right) \\
& \geq-\log (|\mathcal{A}|(C(\beta)+1),
\end{aligned}
$$

where the last line uses inequality (6) (with $l=0$ ), summing both sides over $i \in \mathcal{A}$. Then

$$
\frac{1}{n} L_{n}^{\prime}(\beta, p) \geq \frac{1}{n} H_{n}(\beta, p)-\frac{1}{n} \log (|\mathcal{A}|(C(\beta)+1)),
$$

giving that $\frac{1}{n} L_{n}^{\prime}(\beta, p)$ converges to $H(\beta, p)$ as required.

Norms of random products of matrices are extremely well studied, and so putting our lower bound for $H(\beta, p)$ in the above form may yield useful computations.

We now describe another bound from below on $H(\beta, p)$, which is computationally very simple, and which is sometimes sufficient to conclude that the Hausdorff dimension of $\nu_{\beta, p}$ is 1 .

Proposition 3.6. Suppose that $V_{\beta}$ is finite. Let $\lambda$ be the largest eigenvalue of the matrix $\left((1-p) M_{0}+p M_{1}\right)$. Then

$$
-\log \lambda \leq H(\beta, p),
$$

and hence $\operatorname{dim}_{\mathrm{H}} \nu_{\beta} \geq \min \left\{1, \frac{-\log (\lambda)}{\log (\beta)}\right\}$. 
Proof. We use the norm $\|M\|_{1}=\sum_{i, j}\left|M_{i, j}\right|$. For non-negative matrices $A$ and $B$, we have $\|A\|_{1}+\|B\|_{1}=\|A+B\|_{1}$.

We have

$$
\begin{aligned}
\frac{1}{n} L_{n}^{\prime}(\beta, p) & =-\frac{1}{n} \sum_{a_{1} \cdots a_{n}} m_{p}\left[a_{1} \cdots a_{n}\right] \log \left(\left\|M_{a_{1}} \cdots M_{a_{n}}\right\|\right) \\
& \geq-\frac{1}{n} \sum_{a_{1} \cdots a_{n}} m_{p}\left[a_{1} \cdots a_{n}\right] \log \left(\left\|M_{a_{1}} \cdots M_{a_{n}}\right\|_{1}\right) \\
& \geq-\frac{1}{n} \log \left(\sum_{a_{1} \cdots a_{n}} m_{p}\left[a_{1} \cdots a_{n}\right]\left\|M_{a_{1}} \cdots M_{a_{n}}\right\|_{1}\right) \\
& =-\frac{1}{n} \log \left(\left\|\sum_{a_{1} \cdots a_{n}} m_{p}\left(a_{1} \cdots a_{n}\right) M_{a_{1}} \cdots M_{a_{n}}\right\|_{1}\right) \\
& =-\frac{1}{n} \log \left\|\left((1-p) M_{0}+p M_{1}\right)^{n}\right\|_{1} .
\end{aligned}
$$

By Proposition 3.3, $\frac{1}{n} L_{n}^{\prime}(\beta, p)$ is a lower bound on $H(\beta, p)$ and since

$$
\lim _{n \rightarrow \infty} \frac{1}{n} \log \left\|\left((1-p) M_{0}+p M_{1}\right)^{n}\right\|_{1}=\log \lambda
$$

we have $H(\beta, p) \geq-\log \lambda$.

Since computing eigenvalues is extremely rapid, this approach is the one that we use in practice for proving that $\operatorname{dim}_{\mathrm{H}}\left(\nu_{\beta, p}\right)=1$ for a variety of examples.

\subsection{Computational ideas and examples}

In this section we describe how to use Proposition 3.3 to get explicit bounds on $H(\beta)=H(\beta, 1 / 2)$ and hence on $\operatorname{dim}_{\mathrm{H}} \nu_{\beta}$ for specific examples. For the remainder of the article we concern ourselves only with the case of unbiased Bernoulli convolutions, and no longer include $p$ as a variable.

Suppose $\beta$ is hyperbolic. Then one easily writes a computer program which finds the (finite) graph $\mathcal{G}$ and the matrices $M_{0}$ and $M_{1}$. By Proposition 3.3, we have

$$
\frac{1}{n} L_{n}(\beta) \leq H(\beta) \leq \frac{1}{n} H_{n}(\beta) .
$$


Expressed as a bound on $\operatorname{dim}_{\mathrm{H}} \nu_{\beta}$, it says

$$
\min \left\{1, \frac{1}{n} \frac{L_{n}(\beta)}{\log \beta}\right\} \leq \operatorname{dim}_{\mathrm{H}} \nu_{\beta} \leq \min \left\{1, \frac{1}{n} \frac{H_{n}(\beta)}{\log \beta}\right\} .
$$

Given an $n$ one can, with a computer, calculate numerically the above lower and upper bounds on $H(\beta)$ and $\operatorname{dim}_{\mathrm{H}} \nu_{\beta}$. Unfortunately, the convergence is quite slow, and the computational complexity is high, since evaluating $L_{n}(\beta)$ and $H_{n}(\beta)$ involves summing over $2^{n}$ different sequences.

There is a way to somewhat improve the convergence by pruning the graph $\mathcal{G}$. Call a vertex $x$ redundant if there is no path to $\{0\}$ from $x$ along edges in the graph. Clearly, a vertex is redundant, if and only if all edges from the vertex lead to redundant vertices. We remove all redundant vertices from $\mathcal{G}$ and get a new graph which we denote by $\mathcal{G}^{\prime}$. Using instead this pruned graph to define $\tilde{L}_{n}(\beta)$ in the same way as the definition of $L_{n}^{\prime}(\beta)$, the above bounds on $H(\beta)$ and $\operatorname{dim}_{\mathrm{H}} \nu_{\beta}$ hold with $L_{n}^{\prime}(\beta)$ replaced by $\tilde{L}_{n}(\beta)$.

Example 3.7. To illustrate the above, we let $\beta$ be the largest root of the equation

$$
\beta^{4}-\beta^{3}-\beta^{2}+\beta-1=0 .
$$

Here $\beta \approx 1.5129$ has one other conjugate $\beta^{(2)}$ larger than one in modulus, $\beta^{(2)} \approx-1.1787$. $\beta$ also has two conjugates less than one in modulus, both of which are complex. $\mathcal{G}$ consists of 71 vertices and $\mathcal{G}^{\prime}$ consists of 21 vertices.

Using the graph $\mathcal{G}$ and $n=9$, we find that

$$
\frac{1}{n} \frac{L_{n}^{\prime}(\beta)}{\log \beta}=0.77199 \leq \frac{H(\beta)}{\log \beta} \leq 1.5763=\frac{1}{n} \frac{H_{n}(\beta)}{\log \beta} .
$$

Using instead the pruned graph $\mathcal{G}^{\prime}$ and $n=9$, we find that

$$
\frac{1}{n} \frac{\tilde{L}_{n}(\beta)}{\log \beta}=1.0006 \leq \frac{H(\beta)}{\log \beta} .
$$

We conclude that $\operatorname{dim}_{\mathrm{H}}\left(\nu_{\beta}\right)=1$. We remark that this result does not follow from the aforementioned work of Breuillard and Varjú [4], since in this example

$$
\frac{0.44 \min \left\{\log 2, \log M_{\beta}\right\}}{\log \beta} \approx 0.6146<1
$$


As is illustrated in the above example, even if the upper and lower bounds are far apart, they can still be useful to prove that the Hausdorff dimension is 1 . For the number $\beta$ in the example it is sufficient to take $n=9$ in order to prove that the dimension is 1 . For some other numbers, one needs to take larger values of $n$, resulting in very long computation times.

\subsubsection{Using Proposition 3.6}

We now give some examples to show the advantage of using Proposition 3.6. The key advantage of this proposition lies in the fact that eigenvalues are numerically quick to compute.

Example 3.8. We take $\beta$ as in Example 3.7. Proposition 3.6 gives

$$
\frac{H(\beta)}{\log \beta} \geq 1.3867
$$

and hence $\operatorname{dim}_{\mathrm{H}} \nu_{\beta}=1$.

The lower bound for $H(\beta, p)$ given in Proposition 3.6 is not tight. By looking at Bernoulli convolutions associated with Pisot numbers one can see how far off the true value it is for some examples.

Example 3.9. Let $\beta$ be the Golden ratio. Alexander and Zagier showed that that $\operatorname{dim}_{\mathrm{H}} \nu_{\beta}=0.995570 \ldots$ [3]. Proposition 3.6 gives

$$
\frac{H(\beta)}{\log \beta} \geq 0.9924
$$

and hence $\operatorname{dim}_{\mathrm{H}} \nu_{\beta} \geq 0.9924$.

The fact that the dimension of the Bernoulli convolution in the previous example is known so accurately is due to special properties of the Golden ratio. Outside of a special class of Pisot numbers known as multinacci numbers, there are not many examples of Pisot numbers for which the Hausdorff dimension of $\nu_{\beta}$ was known to three decimal places before the present work, see [16]. See also [8], in which Feng calculated the Hausdorff dimension with high precision for multinacci numbers.

Below, we give an example of what information can be obtained from Proposition 3.6 for Pisot numbers that are not multinacci numbers. 
Example 3.10. Let $\beta$ be the Pisot number given by $\beta^{3}-\beta-1=0$. Since $\beta$ is a Pisot number, we have $\operatorname{dim}_{\mathrm{H}} \nu_{\beta}<1$. Proposition 3.6 gives

$$
\frac{H(\beta)}{\log \beta} \geq 0.99999
$$

Hence $0.99999 \leq \operatorname{dim}_{\mathrm{H}} \nu_{\beta}<1$ and we have obtained the Hausdorff dimension of $\nu_{\beta}$ to five decimal places.

In fact, the numbers in Examples 3.9 and 3.10 already appeared in a work of Lau [19], but not in connection with Hausdorff dimension. The link between the lower bound for $\operatorname{dim}_{\mathrm{H}}\left(\nu_{\beta}\right)$ of our Proposition 3.6 and Lau's Mean Quadratic Variation Dimension are explained in Section 4. We will then see that the lower bound obtained in Example 3.10 follows from Lau's work together with that of Ngai [21].

Finally, we apply our methods to the study of hyperbolic $\beta$ of degree 4 and 5 .

Example 3.11. Let $\beta$ satisfy

$$
a_{5} \beta^{5}+a_{4} \beta^{4}+\cdots+a_{0}=0
$$

with each $a_{i} \in\{-1,0,1\}$. Suppose that $\beta$ is hyperbolic. Then either $\beta$ is Pisot and $\nu_{\beta}$ has Hausdorff dimension less than one, or $\beta$ is not Pisot and $\nu_{\beta}$ has dimension one. The computations are given in Tables 1 and 2, which shows all hyperbolic $\beta$ that are roots of a $\{-1,0,1\}$-polynomial of degree 2 , 3, 4 or 5. Paul Mercat has also done these computations and confirms our results (private communication).

\section{Connections with mean quadratic variation dimension}

In this section, we give an interesting relation between the lower bound $\frac{-\log \lambda}{\log \beta}$ which follows from Proposition 3.6, and another quantity (the so-called mean quadratic variation dimension or $L^{2}$-spectrum) of Bernoulli convolutions. To begin with, let $\mu$ be a Borel probability measure in $\mathbb{R}^{d}$ with compact support. 


\begin{tabular}{r|c|c|c|r} 
Polynomial & $\beta$ & type & lower bound $\left(\frac{-\log \lambda}{\log \beta}\right)$ & size of $\mathcal{G}^{\prime}$ \\
\hline$x^{2}-x-1$ & 1.6180 & Pisot & 0.99240 & 5 \\
\hline$x^{3}-x^{2}-x-1$ & 1.8393 & Pisot & 0.96422 & 7 \\
$x^{3}-x^{2}-1$ & 1.4656 & Pisot & 0.99912 & 49 \\
$x^{3}-x-1$ & 1.3247 & Pisot & 0.99999 & 179 \\
\hline$x^{4}-x^{3}-x^{2}-x-1$ & 1.9276 & Pisot & 0.97333 & 9 \\
$x^{4}-x^{3}-x^{2}+x-1$ & 1.5129 & not Pisot & 1.38670 & 21 \\
$x^{4}-x^{3}-1$ & 1.3803 & Pisot & 0.99999 & 1253 \\
$x^{4}-x^{3}+x^{2}-x-1$ & 1.2906 & not Pisot & 2.50349 & 9 \\
$x^{4}-x^{2}-1$ & 1.2720 & not Pisot & 1.98480 & 25 \\
$x^{4}-x-1$ & 1.2207 & not Pisot & 1.61576 & 1253 \\
$x^{4}+x^{3}-x^{2}-x-1$ & 1.1787 & not Pisot & 3.49147 & 21
\end{tabular}

Table 1: Lower bounds on $\operatorname{dim}_{\mathrm{H}} \nu_{\beta}$ for all hyperbolic $\beta$ of degree 2, 3 and 4 . If the lower bound is larger than one, then $\operatorname{dim}_{\mathrm{H}} \nu_{\beta}=1$.

For $x \in \mathbb{R}^{d}$ and $r>0$, let $B_{r}(x)$ denote the closed ball centered at $x$ of radius $r$. For $q \in \mathbb{R}$, the $L^{q}$-spectrum of $\mu$ is defined as

$$
\tau(\mu, q)=\liminf _{r \rightarrow 0} \frac{\log \Theta_{\mu}(q ; r)}{\log r},
$$

where

$$
\Theta_{\mu}(q ; r)=\sup \sum_{i} \mu\left(B_{r}\left(x_{i}\right)\right)^{q}, \quad r>0, q \in \mathbb{R},
$$

and the supremum is taken over all families of disjoint balls $\left\{B_{r}\left(x_{i}\right)\right\}_{i}$ with $x_{i} \in \operatorname{supp}(\mu)$. In the case $q=2, \tau_{\mu}(2)$ is also called the mean quadratic variation dimension of $\mu$ (see [19]).

By definition, $\tau(\mu, 1)=0$ and $\tau(\mu, q)$ is a concave function of $q$ over $\mathbb{R}$. It follows that $\frac{\tau(\mu, q)}{q-1}$ is monotone decreasing over $(1, \infty)$, and so $\tau^{\prime}(\mu, 1+) \geq$ $\frac{\tau(\mu, q)}{q-1}$ for each $q>1$. In [21], Ngai proved that $\operatorname{dim}_{\mathrm{H}}(\mu) \geq \tau^{\prime}(\mu, 1+)$. As a consequence, $\operatorname{dim}_{\mathrm{H}}(\mu) \geq \frac{\tau(\mu, q)}{q-1}$ for every $q>1$; moreover, the equality $\operatorname{dim}_{\mathrm{H}} \mu=\frac{\tau(\mu, q)}{q-1}$, if it holds for some $q>1$, will imply that $\tau(\mu, \cdot)$ is affine over $[1, q]$.

The concept of $L^{q}$-spectrum plays a fundamental role in multifractal analysis. In [19], Lau computed the $L^{2}$-spectrum of the Bernoulli convolution $\nu_{\beta}$ when $\beta$ is a Pisot number. As pointed out by one of the referees, in the Pisot case 


\begin{tabular}{|c|c|c|c|c|}
\hline Polynomial & $\beta$ & type & lower bound $\left(\frac{-\log \lambda}{\log \beta}\right)$ & size of $\mathcal{G}^{\prime}$ \\
\hline$x^{5}-x^{4}-x^{3}-x^{2}-x-1$ & 1.9659 & Pisot & 0.98357 & 11 \\
\hline$x^{5}-x^{4}-x^{3}-x^{2}-1$ & 1.8885 & Pisot & 0.98227 & 739 \\
\hline$x^{5}-x^{4}-x^{3}-x^{2}+1$ & 1.7785 & Pisot & 0.99576 & 947 \\
\hline$x^{5}-x^{4}-x^{3}-x^{2}+x-1$ & 1.7924 & not Pisot & 1.12741 & 13 \\
\hline$x^{5}-x^{4}-x^{3}-x-1$ & 1.8124 & Pisot & 0.98243 & 349 \\
\hline$x^{5}-x^{4}-x^{3}-x+1$ & 1.6804 & not Pisot & 1.17467 & 139 \\
\hline$x^{5}-x^{4}-x^{3}-1$ & 1.7049 & Pisot & 0.99304 & 339 \\
\hline$x^{5}-x^{4}-x^{3}+x-1$ & 1.5499 & not Pisot & 1.1971 & 1133 \\
\hline$x^{5}-x^{4}-x^{3}+x^{2}-1$ & 1.4432 & Pisot & 0.99998 & 5385 \\
\hline$x^{5}-x^{4}-x^{2}-x-1$ & 1.6851 & not Pisot & 1.1072 & 1265 \\
\hline$x^{5}-x^{4}-x^{2}-x+1$ & 1.5262 & not Pisot & 1.4420 & 139 \\
\hline$x^{5}-x^{4}-x^{2}-1$ & 1.5702 & Pisot & 0.99986 & 841 \\
\hline$x^{5}-x^{4}-x^{2}+x-1$ & 1.4036 & not Pisot & 1.3664 & 1239 \\
\hline$x^{5}-x^{4}-x-1$ & 1.4971 & not Pisot & 1.4216 & 57 \\
\hline$x^{5}-x^{4}+x^{2}-x-1$ & 1.2628 & not Pisot & 2.4946 & 131 \\
\hline$x^{5}-x^{4}+x^{3}-x^{2}-x-1$ & 1.4076 & not Pisot & 1.9447 & 11 \\
\hline$x^{5}-x^{4}+x^{3}-x^{2}-1$ & 1.2499 & not Pisot & 1.8291 & 1617 \\
\hline$x^{5}-x^{4}+x^{3}-x-1$ & 1.2083 & not Pisot & 3.3882 & 11 \\
\hline$x^{5}-x^{3}-x^{2}-x-1$ & 1.5342 & Pisot & 0.99983 & 2635 \\
\hline$x^{5}-x^{3}-x^{2}-x+1$ & 1.3690 & not Pisot & 1.8252 & 947 \\
\hline$x^{5}-x^{3}-x^{2}-1$ & 1.4291 & not Pisot & 1.6106 & 43 \\
\hline$x^{5}-x^{3}-x^{2}+x-1$ & 1.2828 & not Pisot & 2.5554 & 13 \\
\hline$x^{5}-x^{3}-1$ & 1.2365 & not Pisot & 1.4629 & 11173 \\
\hline$x^{5}-x^{3}+x^{2}-x-1$ & 1.2000 & not Pisot & 3.1452 & 947 \\
\hline$x^{5}-x^{2}-1$ & 1.1939 & not Pisot & 1.9356 & 3387 \\
\hline$x^{5}-x-1$ & 1.1673 & not Pisot & 2.2193 & 2807 \\
\hline$x^{5}+x^{3}-x^{2}-x-1$ & 1.1436 & not Pisot & 4.3718 & 97 \\
\hline$x^{5}+x^{4}-x^{3}-x^{2}-1$ & 1.1595 & not Pisot & 4.3005 & 13 \\
\hline$x^{5}+x^{4}-x^{3}-x-1$ & 1.1408 & not Pisot & 4.6298 & 139 \\
\hline$x^{5}+x^{4}-x^{2}-x-1$ & 1.1237 & not Pisot & 2.5707 & 6485 \\
\hline
\end{tabular}

Table 2: Lower bounds on $\operatorname{dim}_{\mathrm{H}} \nu_{\beta}$ for all hyperbolic $\beta$ of degree 5 . If the lower bound is larger than one, then $\operatorname{dim}_{\mathrm{H}} \nu_{\beta}=1$. 
there is certain coincidence between the numerical estimates of our bound $\frac{-\log \lambda}{\log \beta}$ in Tables 1 and 2 and that of the $L^{2}$-spectrum of $\nu_{\beta}$ given by Lau [19]. The following result justifies this connection.

Proposition 4.1. Suppose that $\beta \in(1,2)$ is a Pisot number. Let $\lambda$ be the largest eigenvalue of the matrix $\left((1-p) M_{0}+p M_{1}\right)$. Then

$$
\tau\left(\nu_{\beta, p}, 2\right)=-\frac{\log \lambda}{\log \beta}
$$

Moreover, $\operatorname{dim}_{\mathrm{H}}\left(\nu_{\beta, p}\right)>\frac{\tau\left(\nu_{\beta, p}, q\right)}{q-1}$ for any $q>1$.

Proof. Let $\beta$ be a Pisot number in $(1,2)$. The second part of the proposition follows from the fact that $\tau\left(\nu_{\beta, p}, q\right)$ is strictly concave over the region $(0, \infty)$. This fact comes from some results obtained in [7, 9]. Indeed, by Theorem 5.2 in [7], there exist $\ell, r \in \mathbb{N}$ and a tuple of non-negative $r \times r$ matrices $\left(A_{1}, \ldots, A_{\ell}\right)$ such that $\sum_{i=1}^{\ell} A_{i}$ is irreducible and for any $q>0$,

$$
\tau\left(\nu_{\beta, p}, q\right)=-\frac{P(q)}{\log \beta},
$$

where $P(q):=\lim _{n \rightarrow \infty} \frac{1}{n} \log \sum_{i_{1} \cdots i_{n} \in\{1, \ldots, \ell\}^{n}}\left(\left\|A_{i_{1}} \cdots A_{i_{n}}\right\|_{1}\right)^{q}$. Suppose on the contrary that $\tau\left(\nu_{\beta, p}, \cdot\right)$ is not strictly concave on $(0, \infty)$. Then $\tau\left(\nu_{\beta, p}, \cdot\right)$ is affine on some interval $(a, b)$ with $0<a<b$, and so is the function $P(\cdot)$. This implies that (see the proof of Theorem 1.8 in [9]) $\left(A_{1}, \ldots, A_{\ell}\right)$ has uniform exponent modulo 0 in the sense that there exist $c, t>0$ such that for each $n \in \mathbb{N}$ and any $i_{1} \cdots i_{n} \in\{1, \ldots, \ell\}^{n}$,

$$
\text { either } \quad A_{i_{1}} \ldots A_{i_{n}}=0 \quad \text { or } \quad c^{-1} t^{n} \leq\left\|A_{i_{1}} \cdots A_{i_{n}}\right\|_{1} \leq c t^{n} .
$$

Furthermore, this implies that $\nu_{\beta, p}$ is absolutely continuous (see Theorem 6.2 of [9]), which gives a contradiction since $\nu_{\beta, p}$ is singular $([6])$.

Next we prove the first part of the proposition, i.e. $\tau\left(\nu_{\beta, p}, 2\right)=-\frac{\log \lambda}{\log \beta}$. For this purpose, we first claim that for each $q>0$, there exists a constant $C=C(\beta, p, q)>0$ such that

$$
C^{-1} u_{n}(q) \leq \Theta_{\nu_{\beta, p}}\left(q ; \beta^{-n}\right) \leq C u_{n}(q),
$$

where $\Theta$ is defined as in (7) and

$$
u_{n}(q):=\sum_{a_{1} \cdots a_{n} \in\{0,1\}^{n}} m_{p}\left[a_{1} \cdots a_{n}\right]\left(\left(M_{a_{1}} \cdots M_{a_{n}}\right)_{1,1}\right)^{q-1} .
$$


To prove (8), for each $n \in \mathbb{N}$, we define an equivalence relation $\sim_{n}$ on $\{1, \ldots, \ell\}^{n}$ by

$$
a_{1} \cdots a_{n} \sim_{n} b_{1} \cdots b_{n} \quad \text { if } \quad \sum_{i=1}^{n} a_{i} \beta^{-i}=\sum_{i=1}^{n} b_{i} \beta^{-i},
$$

and let $\Omega_{n}:=\{1, \ldots, \ell\}^{n} / \sim_{n}$ be the corresponding quotient set. Define two maps $S_{0}, S_{1}: \mathbb{R} \rightarrow \mathbb{R}$ by $S_{i}(x)=(x+i) / \beta, i=0,1$. Write for brevity $S_{a_{1} \cdots a_{n}}=S_{a_{1}} \circ \cdots \circ S_{a_{n}}$. It is clear that $S_{a_{1} \cdots a_{n}}=S_{b_{1} \cdots b_{n}}$ if and only if $a_{1} \cdots a_{n} \sim_{n} b_{1} \cdots b_{n}$. Hence by the self-similarity of $\nu_{\beta, p}$,

$\nu_{\beta, p}=\sum_{a_{1} \cdots a_{n} \in\{0,1\}^{n}} m_{p}\left[a_{1} \cdots a_{n}\right] \nu_{\beta, p} \circ S_{a_{1} \cdots a_{n}}^{-1}=\sum_{\left[a_{1} \cdots a_{n}\right] \in \Omega_{n}}\left(M_{a_{1}} \cdots M_{a_{n}}\right)_{1,1} \nu_{\beta, p} \circ S_{a_{1} \cdots a_{n}}^{-1}$,

recalling that $\sum_{b_{1} \cdots b_{n} \in\{0,1\}^{n}: b_{1} \cdots b_{n} \sim_{n} a_{1} \cdots a_{n}} m_{p}\left[b_{1} \cdots b_{n}\right]=\left(M_{a_{1}} \cdots M_{a_{n}}\right)_{1,1}$. Since $\beta$ is a Pisot number, there exists an integer $N$ such that for any $n \in \mathbb{N}$ and any interval $\Delta \subset I:=\operatorname{supp}\left(\nu_{\beta, p}\right)=[0,1 /(\beta-1)]$ of length $\beta^{-n}$, there are at most $N$ many elements $\left[a_{1} \cdots a_{n}\right] \in \Omega_{n}$ such that $S_{a_{1} \cdots a_{n}}(I) \cap \Delta \neq$ $\emptyset$ (see e.g. $[19,7]$ ). Applying this property and (9) one can show (with some additional standard arguments) that $\Theta_{\nu_{\beta, p}}\left(q ; \beta^{-n}\right)$ is comparable to $\sum_{\left[a_{1} \cdots a_{n}\right] \in \Omega_{n}}\left(\left(M_{a_{1}} \cdots M_{a_{n}}\right)_{1,1}\right)^{q}$ (i.e. the ratios between them are uniformly bounded away from 0$)$. We leave the details to the reader. Now it is easy to see that $\sum_{\left[a_{1} \cdots a_{n}\right] \in \Omega_{n}}\left(\left(M_{a_{1}} \cdots M_{a_{n}}\right)_{1,1}\right)^{q}=u_{n}(q)$, and so (8) holds.

In the end, we show that $u_{n}(2)$ is comparable to $v_{n}(2)$, where

$$
v_{n}(2):=\sum_{a_{1} \cdots a_{n} \in\{0,1\}^{n}} m_{p}\left[a_{1} \cdots a_{n}\right]\left\|M_{a_{1}} \cdots M_{a_{n}}\right\|_{1} .
$$

This will conclude our desired result since we have already proved that $\lim _{n \rightarrow \infty}\left(\log v_{n}(2)\right) / n=\log \lambda$ in the proof of Proposition 3.6.

Clearly $v_{n}(2) \geq u_{n}(2)$, and

$$
\begin{aligned}
v_{n}(2) & =\sum_{\left[a_{1} \cdots a_{n}\right] \in \Omega_{n}}\left(M_{a_{1}} \cdots M_{a_{n}}\right)_{1,1}\left\|M_{a_{1}} \cdots M_{a_{n}}\right\|_{1} \\
& \leq \sum_{\left[a_{1} \cdots a_{n}\right] \in \Omega_{n}}\left(\left\|M_{a_{1}} \cdots M_{a_{n}}\right\|_{1}\right)^{2}=: w_{n}(2) .
\end{aligned}
$$

For two elements $\left[b_{1} \cdots b_{n}\right],\left[a_{1} \cdots a_{n}\right] \in \Omega_{n}$, we say that $\left[b_{1} \cdots b_{n}\right]$ is a neighbour of $\left[a_{1} \cdots a_{n}\right]$ if there exist $i, j \in \mathcal{A}$ such that $\beta^{n} x_{i}+\sum_{l=1}^{n}\left(a_{l}-b_{l}\right) \beta^{n-l}=$ 
$x_{j}$. Assume that $n$ is large enough so that $\beta^{n} x_{i}-x_{j} \neq \beta^{n} x_{i^{\prime}}-x_{j^{\prime}}$ when $(i, j) \neq\left(i^{\prime}, j^{\prime}\right)$. Then by the definition (4), we have

$$
\left\|M_{a_{1}} \cdots M_{a_{n}}\right\|_{1}=\sum\left(M_{b_{1}} \cdots M_{b_{n}}\right)_{1,1}
$$

where the sum is over all the neighbours $\left[b_{1} \cdots b_{n}\right]$ of $\left[a_{1} \cdots a_{n}\right]$ in $\Omega_{n}$. Since any element in $\Omega_{n}$ has at most $|\mathcal{A}|^{2}$ many neighbours, and conversely each element in $\Omega_{n}$ is the neighbour of at most $|\mathcal{A}|^{2}$ many elements in $\Omega_{n}$. It follows that

$$
\begin{aligned}
w_{n}(2) & =\sum_{\left[a_{1} \cdots a_{n}\right] \in \Omega_{n}}\left(\left\|M_{a_{1}} \cdots M_{a_{n}}\right\|_{1}\right)^{2} \\
& =\sum_{\left[a_{1} \cdots a_{n}\right] \in \Omega_{n}}\left(\sum_{\substack{\left[b_{1} \cdots b_{n}\right] \in \Omega_{n} \\
\left[b_{1} \cdots b_{n}\right] \text { is a neighbour of }\left[a_{1} \cdots a_{n}\right]}}\left(M_{b_{1}} \cdots M_{b_{n}}\right)_{1,1}\right)^{2} \\
& \leq|\mathcal{A}|^{4} \sum_{\left[a_{1} \cdots a_{n}\right] \in \Omega_{n}}\left(\begin{array}{l}
\sum_{\left[b_{1} \cdots b_{n}\right] \in \Omega_{n}}\left(M_{b_{1}} \cdots b_{n}\right] \text { is a neighbour of }\left[a_{1} \cdots a_{n}\right] \\
\sum_{\left.\left.b_{n}\right)_{1,1}\right)^{2}}
\end{array}\right) \\
& \left.\leq|\mathcal{A}|^{6} \sum_{\left[b_{1} \cdots b_{n}\right] \in \Omega_{n}}\left(M_{b_{1}} \cdots M_{b_{n}}\right)_{1,1}\right)^{2}=|\mathcal{A}|^{6} u_{n}(2) .
\end{aligned}
$$

Hence we obtain that $u_{n}(2) \leq v_{n}(2) \leq w_{n}(2) \leq|\mathcal{A}|^{6} u_{n}(2)$. That is, $u_{n}(2)$ and $v_{n}(2)$ are comparable. It completes the proof of the proposition.

Remark. When $\beta \in(1,2)$ is a hyperbolic algebraic integer, the above proof can be adapted to show that $\tau\left(\nu_{\beta, p}, 2\right) \leq-\frac{\log \lambda}{\log \beta}$.

\section{$5 \quad$ Further Comments}

1. As can be seen from Table 2, when $\beta$ has Galois conjugates close to 1 in modulus the graph $\mathcal{G}$ can be very large. In these cases, calculating the graph $\mathcal{G}$ may not be the most efficient way of proving that $\nu_{\beta}$ has dimension 1 . In a follow up article we show how one can perform counting estimates broadly similar to those of $[15,16]$ on a higher 
dimensional self-affine set with contraction ratios equal to the Galois conjugates of $\beta$. These estimates often yield that $\operatorname{dim}_{\mathrm{H}}\left(\nu_{\beta}\right)=1$, and work even in the case of non-hyperbolic $\beta$.

2. A short argument of Mercat (personal communication) shows that $H(\beta) \leq \log (\beta)$ whenever $\beta$ is a Salem number. Therefore it will hold that $L_{n}(\beta)<\log (\beta)$ for all $n \in \mathbb{N}$ and so our finite time approximation methods will not be able to show that $\operatorname{dim}_{H}\left(\nu_{\beta}\right)=1$ for $\beta$ Salem.

3. According to Lemma 2.4, $V_{\beta}$ is finite if $\beta$ is hyperbolic. Is it true that if $\beta$ is a non-hyperbolic algebraic integer, then $V_{\beta}$ is infinite?

If we in the definition of $V_{\beta}$ only consider $\beta$ and not its conjugates, and define

$$
\begin{aligned}
\tilde{V}_{\beta, n}:=\left\{x=\sum_{i=0}^{n} \epsilon_{i} \beta^{n-i}: \epsilon_{i} \in\{-1,0,1\}\right. \\
\left.\quad \text { and }\left|\sum_{i=0}^{n} \epsilon_{i} \beta^{n-i}\right| \leq \frac{1}{\beta-1}\right\}, \\
\tilde{V}_{\beta}:=\bigcup_{n=0}^{\infty} \tilde{V}_{\beta, n}
\end{aligned}
$$

then, according to a result by Akiyama and Komornik [1], $\tilde{V}_{\beta}$ is finite if and only if $\beta$ is a Pisot number.

\section{Acknowledgments}

We would like to thank the referees for their helpful comments, and thank Pierre Arnoux and Paul Mercat for many helpful conversations.

The majority of this work took place as research in pairs at the Centre International de Recontres Mathématiques in Luminy during a Research in Pairs program. We would like to thank the CIRM and the Jean-Morlet semester "Tiling and discrete geometry" for financial and logistical support. S. Akiyama was also supported by Japan Society for the Promotion of Science grants (17K05159, 17H02849). D.-J. Feng, T. Kempton and T. Persson are also grateful to Institut Mittag-Leffler in Djursholm and the semester 
"Fractal geometry and dynamics" during which parts of this work were done. D.-J. Feng was also partially supported by the Hong Kong Research Grants Council General Research Funds grants (projects CUHK2130445, CUHK2130546).

\section{References}

[1] S. Akiyama, V. Komornik. Discrete spectra and Pisot numbers. J. Number Theory, 133 (2013), no. 2, 375-390.

[2] S. Akiyama, J. M. Thuswaldner, and T. Zaïmi. Characterisation of the numbers which satisfy the height reducing property. Indag. Math. (N.S.), 26(1):24-27, 2015.

[3] J. C. Alexander and D. Zagier. The entropy of a certain infinitely convolved Bernoulli measure. J. London Math. Soc. (2), 44(1):121-134, 1991.

[4] E. Breuillard and P. P. Varjú. Entropy of Bernoulli convolutions and uniform exponential growth for linear groups. ArXiv preprint, arXiv:1510.04043v3, 2015. To appear in J. Anal. Math.

[5] E. Breuillard and P. P. Varjú. On the dimension of Bernoulli convolutions. arXiv preprint, arXiv:1610.09154, 2016.

[6] P. Erdős. On a family of symmetric Bernoulli convolutions. Amer. J. Math., 61:974-976, 1939.

[7] D.-J. Feng, Smoothness of the $L^{q}$-spectrum of self-similar measures with overlaps. J. London Math. Soc. (2), 68(1): 102-118, 2003.

[8] D.-J. Feng. The limited Rademacher functions and Bernoulli convolutions associated with Pisot numbers. Adv. Math., 195(1):24-101, 2005.

[9] D.-J. Feng, C.-H. Lo and S. Shen. Lyapunov exponents for products of matrices. arXiv preprint, arXiv:1702.07251, 2017.

[10] D.-J. Feng and N. Sidorov. Growth rate for beta-expansions. Monatsh. Math., 162(1):41-60, 2011. 
[11] C. Frougny. Representations of numbers and finite automata. Math. Systems Theory, 25(1):37-60, 1992.

[12] H. Furstenberg and H. Kesten. Products of random matrices. Ann. Math. Statist., 31:457-469, 1960.

[13] A. M. Garsia. Arithmetic properties of Bernoulli convolutions. Trans. Amer. Math. Soc., 102:409-432, 1962.

[14] A. M. Garsia. Entropy and singularity of infinite convolutions. Pacific J. Math., 13:1159-1169, 1963.

[15] K. G. Hare and N. Sidorov. A lower bound for Garsia's entropy for certain Bernoulli convolutions. LMS J. Comput. Math., 13:130-143, 2010.

[16] K. G. Hare and N. Sidorov. A lower bound for the dimension of Bernoulli convolutions. to appear in Experimental Mathematics, https://doi.org/10.1080/10586458.2017.1301841

[17] M. Hochman. On self-similar sets with overlaps and inverse theorems for entropy. Ann. of Math. (2), 140(2):773-822, 2014.

[18] M. Hochman and P. Shmerkin. Local entropy averages and projections of fractal measures. Ann. of Math. (2), 175(3): 1001-1059, 2012.

[19] K.-S. Lau. Dimension of a family of singular Bernoulli convolutions. J. Funct. Anal., 116(2): 335-358, 1993.

[20] P. Mercat. Semi-groupes fortement automatiques. Bull. Soc. Math. France, 141(3):423-479, 2013.

[21] S.-M. Ngai, A dimension result arising from the $L^{q}$-spectrum of a measure. Proc. Amer. Math. Soc., 125(10): 2943-2951, 1997.

[22] P. Shmerkin. On the exceptional set for absolute continuity of Bernoulli convolutions. Geom. Funct. Anal., 24(3):946-958, 2014.

[23] B. Solomyak. On the random series $\sum \pm \lambda^{n}$ (an Erdös problem). Ann. of Math. (2), 142(3):611-625, 1995.

[24] P. P. Varjú. Absolute continuity of Bernoulli convolutions for algebraic parameters. ArXiv preprint, arXiv:1602.00261, 2016. 
[25] P. P. Varjú. Recent progress on Bernoulli convolutions. ArXiv preprints, arXiv:1608.04210, 2016.

[26] P. Walters, An introduction to ergodic theory. Graduate Texts in Mathematics, 79. Springer-Verlag, New York-Berlin, 1982. 